\title{
Memory for the pragmatic implications of sentences
}

\author{
WILLIAM F. BREWER \\ University of Illinois al Lirhana-Champaign, Champaign, Illinois 61820
}

\begin{abstract}
A sentence pragmatically implies another sentence when information in the first sentence leads the hearer to expect something that is neither explicitly stated nor necessarily implied by the original sentence. Thus, The safe-cracker put the match to the fuse pragmatically implies The safe-cracker lit the fuse. In a cued recall task with sentences containing pragmatic implications, $19 \%$ of the items were recalled correctly while $26 \%$ of the responses consisted of the pragmatic implications of the original sentences. The data were interpreted as demonstrating the strong interaction of the subjects' long-term knowledge with the episodic memory for sentences task.
\end{abstract}

The purpose of the present paper is threefold: (1) to propose a classification of the heterogeneous types of "inferences" used in recent memory experiments, (2) to show the effects in recall of one of these classes-the class of pragmatic implications, and (3) to discuss the theoretical implications of the findings.

Recent experiments on inferences in the comprehension and memory of linguistic material have used a great variety of types of inferences. Typical examples are: There is a tree with a box beside it, and a chair is on top of the box. The box is to the right of the tree implies The chair is to the right of the tree (Bransford, Barclay, \& Franks, 1972). The bear was smarter than the hawk, the hawk was smarter than the wolf implies The bear was smarter than the wolf (Potts, 1972). He was pounding the nail implies He was using the hammer (Johnson, Bransford, \& Solomon, 1973). Miss America said that she played the tuba implies Miss America played the tuba (Harris, 1974). The fuse on the dynamite was not long implies The fuse on the dynamite was short (Brewer \& Lichtenstein, 1975). The English professor told his students a dull story about Jane Austin implies The English professor bored his students with a story about Jane Austin (Schweller, Brewer, \& Dahl, 1976). It is obvious that these types of inferences differ on a wide variety of dimensions; however, one way of organizing them is to distinguish between logical implication and pragmatic implication.

Recent work in linguistics has uncovered a number of interesting semantic relationships between sentences, some of which involve the implications of sentences. The term "logical implication" will be used to refer

The author wishes to thank Kay Bock, Ellen Brewer, Richard Harris, Ken Schweller, and Tom Thieman for help in various stages of this experiment. Requests for reprints should be sent to William F. Brewer, Department of Psychology, University of Illinois, Psychology Building, Champaign, Illinois 61820 . to those semantic relationships where one sentence seems to be necessarily implied by another. Thus, (1) I regret that Russell made the mistake logically implies I believe that Russell made the mistake (cf. Kiparsky \& Kiparsky, 1970). (2) Burrhus is taller than Noam logically implies Noam is shorter than Burrhus (cf. Katz, 1972). (3) The psychologist managed to teach the rat to talk logically implies The psychologist taught the rat to talk (cf. Karttunen, 1971).

The term "pragmatic implication" will be used to refer to a different relationship between sentences. A sentence pragmatically implies another sentence when the information in the first sentence leads the hearer to expect something neither explicitly stated nor necessarily implied in the first sentence. Thus, The karate champion hit the cinder block pragmatically implies The karate champion broke the cinder block. Similarly, Mr. Roberts says it is raining outside prag. matically implies $I t$ is raining outside. For ease of exposition, the pragmatic implication will be represented by a particular sentence, although it is assumed that the underlying implication is nonlinguistic and could be expressed by a variety of different sentences. While the concept of pragmatic implication has been developed here to account for certain types of errors in sentence recall, several philosophers (Bar-Hillel, 1946; Black, 1958) have developed a similar concept for philosophical purposes.

It is possible to use the conjunction but to provide a very sensitive test for pragmatic implication. Lakoff (1971) has pointed out that but is frequently used in a "denial-of-expectation" sense. For example, in the sentence, $A$ platypus is a mammal but it lays eggs, the word but is used to indicate that the information in the second clause is contrary to the expectations produced by the information in the first clause. Therefore, but in the denial-of-expectation sense can be used as a test of pragmatic implication. If one sentence (or abstract proposition) pragmatically implies 
another, negating the second statement and conjoining the two sentences with but should produce an acceptable sentence. Thus, to see if The hungry python caught the mouse pragmatically implies The hungry pythom ate the mouse, the second sentence is negated and conjoined with the first to give The hungry python caught the mouse but did not eat it (an acceptable sentence).

It appears that the but not test is relatively specific for the relation of pragmatic implication. The test excludes semantically synonymous sentences ( ${ }^{*} T h e$ hungry python caught the mouse but the mouse was not caught by the hungry python). It also excludes logically implied sentence pairs such as $(1)^{*} I$ regretted that the python ate the mouse but the python did not eat the mouse (factive relation). (2) *The python was longer than the garter snake but the garter snake was not shorter than the python (entailment). (3) *The python managed to eat the mouse but the python did not eat the mouse (implicative relation). And finally, the but not test excludes sentence pairs that have no apparent relation to each other $\left({ }^{*}\right.$ The hungry python caught the mouse but the specific gravity of copper is not 8.9). A more complete and detailed discussion of pragmatic implication can be found in Harris and Monaco (in press).

Psychologists have found the study of implications in comprehension and memory to be important because of the constraints they place on models of the comprehension and memory processes. Models of memory that suggest that memory is a passive system which registers the words or literal meanings of linguistic input have considerable difficulty in dealing with the finding that subjects' recall of linguistic material includes inferences drawn from the original material (cf. Bransford \& McCarrell, 1974, for a discussion).

The importance of inferences for models of memory has been noted from the very beginning of experimental studies on the topic. In 1894, Binet and Henri made a qualitative analysis of children's recall of prose and pointed out what they called "errors of imagination" (cf. Thieman \& Brewer, in press). Bartlett (1932) also made a qualitative examination of recall protocols and noted that the subjects' knowledge and beliefs about the original material were reflected in the protocols. Neither of these studies had much impact on modern memory research, so it was the classic study of Bransford, Barclay, and Franks (1972) that revived theoretical interest in the issue. Using a recognition memory procedure, these researchers showed that subjects confuse sentences such as Three turtles rested on a floating $\log$ and a fish swam beneath them with sentences such as Three turtles rested on a floating $\log$ and a fish swam beneath it. On the basis of this data, Bransford et al. attacked linguistic theories of sentence menory and proposed that in sentence memory tasks, subjects use the linguistic information and their previous knowledge to construct new semantic descriptions for recall.

A number of studies have followed up the findings of Bransford et al. (1972) using a wide variety of inference types. Using the distinction between logical and pragmatic implication presented above, it is possible to classify the studies into several broad groups. The studies by Bransford et al. (1972), Paris and Carter (1973), and Potts (1972) all used logical implications and recognition memory procedures. They all found that subjects made false recognition responses to the logical implications of the original sentences. The study by Johnson et al. (1973) and the experiment dealing with perlocutionary speech acts by Schweller et al. (1976) used pragmatic implications and recognition memory procedures. Both of these studies found that subjects made false recognition responses to the pragmatic implications of the original sentences. The studies by Brewer and Lichtenstein (1975) and Harris (1974) compared logical and pragmatic implications. Brewer and Lichtenstein used recall procedures and found that subjects gave both logical and pragmatic implications in recall. The study by Harris (1974) was particularly interesting in that he compared logical implications (John managed to finish the job implies John finished the job) with pragmatic implications (John suid he finished the job implies John finished the job) on both a comprehension task and a memory task. On the comprehension task, the subjects showed a clear discrimination between logical implications and pragmatic implications. For the logical implication pairs, the subjects rated the logical implication true, given the original sentence. For the pragmatic implication pairs, they rated the truth of the pragmatic implications indeterminant, given the original sentence. Yet, when the same materials were used in the memory task, the subjects made false recognition responses to both logical implications and pragmatic implications. Two additional studies that have dealt with inferences in memory tasks are Kintsch (1974) and Moeser (1976). In both of these studies, it appears that the phenomena being studied are aspects of text cohesiveness (cf. Halliday \& Hasan, 1976) and thus the inferences would best be dealt with in the framework of studies on the memory and comprehension of cohesion in text.

Overall, the results of studies on inferences in comprehension and memory seem quite clear. In a recognition task, subjects will make false recognition responses to both logical implications and pragmatic implications of the original material, even though the two types of inferences can be shown to differ in comprehension tasks (cf. Harris, 1974). The finding that subjects make false recognition responses to pragmatic implications is particularly surprising. The subjects are clearly showing an active processing of the material they are exposed to, since they give false recognition responses to sentences that are only a 
pragmatically possible outcome of the events described in the original sentence.

While there is considerable agreement in these recent studies, there are some restrictions on the findings. Most of the findings that have been interpreted as examples of pragmatic implication were taken from studies that were directed at slightly different issues, and so used somewhat narrow types of pragmatic implications, such as perlocutionary speech acts (Schweller et al., 1976), factive and nonfactive verbs (Harris, 1974), instruments and consequences (Johnson et al., 1973), sentences with continuous and dichotomous antonyms (Brewer \& Lichtenstein, 1975). In addition, there has been a general reliance on recognition memory tasks. In order to carry out a recognition memory study, the experimenter has to select the implication to be tested, yet, due to the creative nature of the comprehension process, it may not make sense to pick one particular implication as the implication derivable from a given sentence. In addition, the experimenter has to select a particular surface form for the implication and for the foils used in the task. Thus, the results may be distorted by the interaction of the subjects' memory of the original surface forms and the particular surface forms chosen by the experimenter for the recognition task. And, finally, it seems somewhat incongruous for theorists who favor reconstructive models of memory to present subjects with sentences to be recognized rather than to allow the subjects to reconstruct their responses. Given these limitations of the previous studies, the present experiment was designed to focus on the theoretically interesting class of pragmatic implications, study these implications in a recall paradigm, and cover a wide range of types of pragmatic implication.

\section{METHOD}

\section{Subjects}

The subjects were 25 undergraduate students at the University of Illinois who participated in the experiment as part of a course requirement in introductory psychology.

\footnotetext{
Materials

The experimental materials consisted of 46 implication sentences and 46 filler sentences. The original pool of implication sentences was developed by attempting to write sentences that would lead the hearer to expect something that was neither explicitly stated nor necessarily implied by the sentence. Thus, someone hearing The safecracker put the match to the fuse might expect that the fuse ignited, even though this fact is not contained in the literal meaning of the sentence, nor logically implied by it. In order to show the generality of the phenomenon, an attempt was made to make the items as heterogeneous as possible with respect to the knowledge that would lead the hearer to make a particular inference. For example, to make the inference that the doorbell rang upon hearing the sentence, The narcotics officer pushed the doorbell, requires that the hearer know that doorbells typically ring when pushed, while to make the inference that the python ate the mouse upon hearing the sentence, The hungry python caught the mouse, requires that the hearer know that hungry pythons eat mice.
}

In order to insure that the implication sentences did give rise to the predicted inferences, they were examined with the but not test. All implication sentences used in the present experiment met the but not test, in that an acceptable sentence resulted from the conjunction of the original sentence and the negation of its predicted implication. The 46 filler sentences were sentences that did not contain obvious pragmatic implications. The order of the 92 sentences used in the present experiment was randomized, and the resulting master list was subdivided into four experimental lists of 23 sentences each.

\section{Procedure}

The subjects were told that they were participating in a study of memory for sentences. The instructions stated that written recall would be required, but did not explicitly suggest either a rote-memory or a gist-memory strategy. Each subject received all four 23-item lists. Subjects were seen in small groups of four or five. The order of the experimental lists was counterbalanced. The experimenter read each list of sentences aloud with normal intonation, allowing $2 \mathrm{sec}$ between sentences. After the last sentence on each list, the subjects were given cued recall tests for three of the filler sentences from that list. The recall cues consisted of the initial noun phrases of the sentences. The subjects were given $40 \mathrm{sec}$ to write the three sentences. After they had completed the cued recall test for the three filler sentences on the final list, the subjects were given a cued recall test for the 46 implication sentences and the 34 filler sentences that had not been tested previously. The recall cues consisted of the initial noun phrases of the sentences. The subjects were given 16 min to write their answers.

\section{RESULTS}

The implication sentences from the recall protocols were scored using three different sets of criteria: strict, standard, and liberal. (1) With the strict criteria, each response was classified as correct, implication, omit, or error. A sentence was scored as correct if it was written exactly as presented. An implication was scored if the response written by the subject met the but not test when conjoined with the original sentence and what remained of the original sentence met the criteria for the correct category. An omit was scored when nothing was written after the recall cue. All other responses were scored as errors. (2) With the standard criteria, each item was also scored as correct, implication, omit, or error. However, an item was scored as correct as long as it preserved the meaning of the original sentence. Thus, synonym substitutions, determiner shifts, and optional syntactic transformations were ignored. An implication was scored if the response written by the subject met the but not test when conjoined with the original sentence and what remained of the original sentence met the criteria for the correct category. An omit was scored when nothing was written. All other responses were scored as errors. (3) For the liberal criteria, the responses were scored as they were for the standard criteria except that every response that met the but not test was scored as an implication even if the sentence contained an error of some type.

Under the strict scoring criteria, the results for the implication sentences were: correct, $11.5 \%$; omit, $34.3 \%$; implication, 20.5\%; error, $33.7 \%$. Under the standard scoring criteria, the results were: correct, $19.1 \%$; omit, 
$34.3 \%$; implication, $26.4 \%$; error, $20.1 \%$. Under the liberal scoring criteria, the results were: correct, $19.1 \%$; omit, $34.3 \%$; implication, $30.6 \%$; error, $15.9 \%$. Across all 46 implication sentences, the percentage of implications ranged from $0 \%$ to $80 \%$ for strict scoring and from $4 \%$ to $88 \%$ for both standard and liberal scoring. Table 1 gives the modal implication response, the number of correct responses, and the number of implication responses for 10 of the sentences. The results are clear-cut: Subjects do frequently recall the pragmatic implication of a sentence instead of the sentence itself. With carefully designed materials, the effect is very strong. For 26 of the 46 sentences used in this experiment, there were more implications than correct responses (standard scoring criteria).

Qualitative examination of the responses reveals a number of interesting phenomena. For some of the sentences, there was a single obvious surface form that expressed the implication contained in the original sentence. For example, all of the implication responses produced by the narcotics officer pushed the doorbell were in the same form, The narcotics officer rang the doorbell. However, for other sentences, there was no single obvious surface form that captured the inference

Table 1

Modal Implication Responses, Number of Correct Responses, and Number of Implication Responses for 10 Sentences

Sentence: The paratrooper leaped out the door. (5)

Implication: The paratrooper jumped out of the plane. (7)

Sentence: The safecracker put the match to the fuse. ( 0 )

Implication: The safecracker lit the fuse.

Sentence: The clumsy chemist had acid on his coat. Implication: The clumsy chemist spilled acid on his coat.

Sentence: The narcotics officer pushed the doorbell. Implication: The narcotics officer rang the doorbell.

Sentence: The hungry python caught the mouse. Implication: The hungry python ate the mouse.

Sentence: The angry rioter threw the rock at the window.

Implication: The angry rioter threw a rock through the window.

Sentence: The absent-minded professor didn't have his car keys.

Implication: The absent-minded professor forgot his car keys.

Sentence: Dennis the Menace sat in Santa's chair and asked for an elephant.

Implication: Dennis the Menace sat in Santa's lap and asked for an elephant.

Sentence: The flimsy shelf weakened under the weight of the books.

Implication: The flimsy shelf collapsed under the weight of the books.

(4)

Sentence: The fireman sprayed water on the fire. (4) Implication: The firemen put out the fire.

(4)

Note--The figure following the original sentence is the number of correct responses for that item, and the figure following the modal implication is the number of implication responses for the same item (standard scoring criteria). produced by the original sentence, and the implication responses varied widely in surface expression. Thus, the implication responses for the crucial verb in The Sherman tank hit the flimsy roadblock included plowed through, went through, crashed through, broke through, drove through, and went right through.

Since the number of implication responses varied widely across sentences, an attempt was made to examine some of the factors contributing to this variability. In making an implication response, it would appear that there should be a tradeoff between retained surface structure and making the inference. It should be harder to make the error of recalling the implication when the inference requires the deletion of much surface information than when it does not. Thus, there should be fewer implication responses for sentences such as The POW put his pen to the confession (implication: The POW signed his name to the confession-three words deleted) than for sentences such as The barnacles were growing on the ship (implication: The barnacles were growing on the side of the ship-no words deleted). Also, it would seem that there might be a tradeoff between the number of new words required to express the inference and the number of implication responses. Thus, there should be fewer implication responses for sentences such as The mayor opened the new super highway with gold scissors (implication: The mayor opened the new super highway by cutting the ribbon with gold scissors-four words added) than for sentences such as The clumsy chemist had acid on his coat (implication: The clumsy chemist spilled acid on his coat-one word added).

In order to test these hypotheses, three scores were developed for each of the 46 sentences: (1) deletion score, the number of words that must be deleted from the original sentence to produce the modal implication response; (2) addition score, the number of words that must be added to the original sentence to produce the modal implication response; (3) total score, the sum of the deletion score and the addition score. These scores were correlated with the number of implication responses under the standard scoring criteria. The correlations were: deletion score, -.05 ; addition score, $-.41(\mathrm{p}<.005)$; and total score, $-.29(\mathrm{p}<.05)$. Thus, the amount of surface information that must be discarded does not appear to be a factor in the occurrence of an implication response, while the amount of new surface information that must be added is important in determining whether an implication response will occur.

Perhaps the most interesting finding revealed in the qualitative analysis of the data is the occurrence of ambiguous implications. For some of the sentences, the exact nature of the implication contained in the original sentence was ambiguous, and so different subjects made different inferences. Thus, the verb phrase of the sentence, The absent-minded professor 
didn't have his car keys was recalled as: forgot his car keys, lost his car keys, and left his keys in the car. In a similar fashion, the verb in the sentence, The flimsy shelf weakened under the weight of the books was recalled as: bent, swayed, gave way, broke, collapsed, and fell. These responses give a good qualitative indication of the types of inferential processes going on and clearly show that pragmatic implications are not fully determined by the original sentence and are thus quite different from logical implications.

\section{DISCUSSION}

Perhaps the most interesting aspect of the results of this experiment is the robust nature of the recall errors in the standard laboratory setting. For strong sentences such as those listed in Table 1, the subject is far more likely to write down the pragmatic implication of the original sentence than he is to write the original sentence itself. This seems a most unlikely finding if the experiment is examined from the subject's point of view. The subject comes into a room and is asked to memorize a list of unconnected sentences. The subject hears a sentence such as The hungry python caught the mouse. The subject knows that there is no such hungry python and that the event never occurred. The subject's task in the experiment is to learn the sentences as useless bits of episodic information, and not to update his or her long-term knowledge of pythons. Yet the recall protocols show that the subject's long-term knowledge that hungry pythons are likely to eat mice interacts with the episodic information the subject hears to produce the pragmatic implication in recall that The hungry python ate the mouse.

A number of investigators have suggested that subjects receiving isolated lists of sentences with instructions to memorize the material will not deeply process or elaborate the material (Barclay, 1973). The results of the present study and previous work (Schweller et a1., 1976) suggest just the opposite. The subject's long-term knowledge of the world is so intimately related to the language comprehension and memory systems that it is brought to bear even in a situation as "unreal" as the laboratory list-learning setting. This view is also supported by the resistance of the effect to modification by instructions. Informal studies have shown that even severe rote-memory instructions ("try to be a tape recorder") have little effect in reducing the number of pragmatic inferences in recall.

Due to the strength of the effect and its intuitive appeal as an example of reconstructive memory, the recall of items such as those in Table 1 makes a good laboratory demonstration. The inferences appear to be quite automatic and nondeliberate on the part of the subjects. In fact, after carrying out the experiment as a demonstration and finding that $90 \%$ of the class have written the same "wrong" sentence, the class may refuse to believe that the experimenter really read the original sentence.

While the present experiment is a good demonstration of the reconstruction of pragmatic implications in recall, it is clear that much more work is needed to account for the details of the recall process. In particular, the extreme differences in the strength of the effect across items needs to be accounted for. It would seem that the particular findings for a given item are the result of a combination of the amount of original surface information retained, the strength of the implication, the interaction of the episodic information provided by the sentence with the relevant knowledge in long-term memory, and the possible surface forms available to the subject to express the resulting information.

\section{REFERENCES}

BAR-HILLEL, Y. Analysis of "correct" language. Mind, 1946, 55 , 328-340. (Reprinted in Y. Bar-Hillel, Aspects of language. Amsterdam: North-Holland, 1970.)

BARCLAY, J. R. The role of comprehension in remembering sentences. Cognitive Psychology, 1973, 4, 229-254.

Bartlett. F. C. Remembering. London: Cambridge University Press, 1932.

BLACK. M. Presupposition and implication. In S. Uyeda (Ed.), $A$ way to the philosophy of science. Tokyo: Waseda University Press, 1958. (Reprinted in M. Black, Models and metaphors. Ithaca: Cornell, 1962.)

Bransford, J. D., Barclay, J. R., \& Franks, J. J. Sentence memory: A constructive versus interpretive approach. Cognitive Psychology, 1972, 3, 193-209.

BRANSFORD, J. D., \& MCCARRELL, N. S. A sketch of a cognitive approach to comprehension: Some thoughts about understanding what it means to comprehend. In W. B. Weimer \& D. S. Palermo (Eds.), Cognition and the symbolic processes. Hillsdale, N.J: Erlbaum, 1974.

BREWER, W. F., \& LICHTENSTEIN, E. H. Recall of logical and pragmatic implications in sentences with dichotomous and continuous antonyms. Memory \& Cognition, 1975, 3, 315-318.

Halliday, M. A. K., \& Hasan, R. Cohesion in English. London: Longman, 1976.

HARRIs, R. J. Memory and comprehension of implications and inferences of complex sentences. Journal of Verbal Learning and Verbal Behavior, 1974, 13, 626-637.

HaRRIs, R. J., \& MONACo, G. E. The psychology of pragmatic implication: Information processing between the lines. Journal of Experimental Psychology: General, in press.

Johnson, M. K., Bransford, J. D., \& Solomon, S. K. Memory for tacit implications of sentences. Journal of Experimental Psychology, 1973, 98, 203-205.

KaRTrUNEN, L. Implicative verbs. Language, 1971, 47, 340-358.

Katz, J. J. Semantic theory. New York: Harper \& Row, 1972.

KInTsCH, W. The representation of meaning in memory. Hillsdale, N.J: Erlbaum, 1974. 
Kiparsxxy, P., \& Kiparsky, C. Fact. In M. Bierwisch \& K. E. Heidolph (Eds.), Progress in linguistics. The Hague: Mouton, 1970.

LAxofF, R. If's, and's, and but's about conjunction. In C. J. Fillmore \& D. T. Langendoen (Eds.), Studies in linguistic semantics. New York: Holt, Rinehart, \& Winston, 1971.

MoEser, S. D. Inferential reasoning in episodic memory. Journal of Verbal Learning and Verbal Behavior, 1976, 15, 193-212.

Parus, S. G., \& Carter, A. Y. Semantic and constructive aspects of sentence memory in children. Developmental Psychology, 1973, 9, 109-113.
Potrs, G. R. Information processing strategies used in the encoding of linear orderings. Joumal of Verbal Learning and Verbal Behavior, 1972, 11, 727-740.

Schweller, K. G., Brewer, W. F., \& Dahi, D. A. Memory for illocutionary forces and perlocutionary effects of utterances. Journal of Verbal Leaming and Verbal Behavior, 1976, 15, 325-337.

Thieman, T. J., \& Brewer, W. F. Alfred Binet on memory for ideas. Genetic Psychology Monographs, in press.

(Received for publication February 8, 1977; revision accepted September 9,1977 .) 